\title{
Carnets
}

Revue électronique d'études françaises de l'APEF

Première Série - 2 Numéro Spécial | 2010

Littératures nationales: suite ou fin. Résistances, mutations \& lignes de fuite

\section{Entre regionalisme et inter-culturalisme : A la recherche de la poésie acadienne contemporaine (des années 1970 à de nos jours)}

\section{Gwénaël Lamarque}

\section{(2) OpenEdition}

Journals

Édition électronique

URL : http://journals.openedition.org/carnets/4811

DOI : 10.4000/carnets.4811

ISSN : 1646-7698

Éditeur

APEF

Édition imprimée

Date de publication : 1 juin 2010

Pagination : 15-26

\section{Référence électronique}

Gwénaël Lamarque, « Entre regionalisme et inter-culturalisme : A la recherche de la poésie acadienne contemporaine (des années 1970 à de nos jours) », Carnets [En ligne], Première Série - 2 Numéro Spécial | 2010, mis en ligne le 16 juin 2018, consulté le 27 avril 2019. URL : http:// journals.openedition.org/carnets/4811; DOI : 10.4000/carnets.4811

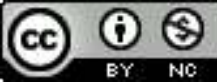

Carnets est mis à disposition selon les termes de la licence Creative Commons - Atribution - Pas d'utilisation commerciale 4.0 International. 


\section{ENTRE REGIONALISME ET INTER-CULTURALISME \\ A la recherche de la poésie acadienne contemporaine (des années 1970 à de nos jours)}

GWENAËL LAMARQUE

EA 2958 - CEMMC - Université de Bordeaux 3 recherche.francophonie@laposte.net

\section{Résumé}

Parmi les formes d'expression littéraires possibles, la poésie occupe une place de choix spécifiquement en Acadie. À en croire son dynamisme, elle se hisse comme l'une des institutions de la culture acadienne, les premiers écrivains acadiens étant avant tout des poètes. Cet article se propose de discuter la notion de "littérature nationale", au regard de cette production littéraire particulière.

\section{Abstract}

Among the literary practices, poetry, because of its aesthetic significance, occupies a special place, especially in Acadia. As its dynamism leads one to believe, it has raised itself to the position of an institution of Acadian culture, the first Acadian writers being above all poets. This article thus will discuss the notion of a "national literature" with regard to this practice in particular.

Mots-clés: Acadie, poésie, américanité, acadienité, littérature, francophonie, Amériques Keywords: Acadia, acadianity, americanity, Americas, literature, French-speaking world, poem 
La francophonie des Amériques est riche de ses diversités. À côté de la très rayonnante et médiatique société québécoise, coexistent d'autres communautés francophones, qualifiées un peu sommairement de "minoritaires" ou de francophonies "hors Québec". Parmi elles, la société acadienne occupe une plage privilégiée. Premiers colons à s'être installés en Amérique en 1604 pour y fonder une colonie de peuplement, les Acadiens sont les victimes des contentieux franco-britanniques et sont méthodiquement déportés au milieu du XVIII ème siècle. Dispersés aujourd'hui aux quatre coins du monde, ils vivent principalement dans les Provinces Maritimes du Canada, mais aussi en Louisiane (EtatsUnis). II s'agit donc d'une diaspora francophone évoluant dans un milieu majoritairement anglophone, ne bénéficiant d'aucune structure de soutient étatique à la différence par exemple des Québécois.

Souvent méconnue, la poésie acadienne s'est imposée comme un champ d'étude parfaitement pertinent dans la perspective de ce colloque autour des littératures nationales. Tout d'abord, parce qu'il s'agit d'une véritable institution au sein même de la culture acadienne: les premiers écrivains acadiens étaient effectivement avant tout des poètes comme Napoléon Landry ou François-Moise Lanteigne. Ensuite parce qu'elle fait preuve d'un dynamisme ininterrompu que consacre notamment la publication cet été même d'une anthologie de la poésie acadienne par Serge Patrice Thibodeau.

C'est effectivement à travers elle que s'exprime le mieux et le plus naturellement l'âme et la sensibilité acadienne. Enfin, parce que cette vitalité poétique est largement due à l'existence de deux grandes maisons d'édition: les éditions d'Acadie et les éditions PerceNeige. La première est lancée en 1972 avec pour objectif de publier l'ensemble des auteurs acadiens, écrivains, dramaturges et poètes. La seconde est fondée un peu plus tard en décembre 1980 avec le statut de maison d'édition sans but lucratif, subventionnée par le Conseil des Arts du Canada et la Direction du Développement des Arts du NouveauBrunswick. Contrairement à la première, elle consacre une part beaucoup plus importante de ses publications aux récits poétiques. Agissant telle une coopérative de jeunes poètes, avant que Gérald LeBlanc n'en assure la direction littéraire en 1990, cette maison a publié la quasitotalité des œuvres des poètes acadiens de ces trente dernières années.

Alors, en quoi la poésie acadienne contemporaine se détache-t-elle de ses sœurs francophones et ses voisines américaines? En quoi est-elle "nationale"? Quelle conscience identitaire véhicule-t-elle? Est-t-elle - en raison de sa géopolitique particulière - le prototype d'une littérature régionale ou plutôt d'une "littérature monde" pour reprendre la typologie proposée dans le cadre de cette rencontre? Doit-on être d'accord avec Serge Patrice Thibodeau, lorsqu'il affirme: 
On observe entre autres dans cette poésie le phénomène identitaire et ses dérives; le temps cyclique et les quatre saisons (...); le rapport à la langue et les variantes du français (...); l'usage baroque des symboles de la foi catholique romaine; le profond sentiment d'appartenance à l'Amérique; la convivialité avec la langue anglaise et les langues étrangères; les pôles contradictoires que sont l'appel de la route et les gestes banals du quotidien; (...) une forme d'autodérision débridée $(\ldots)^{1}$ ?

Au fond, la poésie acadienne ressemble à la littérature acadienne de manière plus générale (Lamarque, 2008a) en se construisant dans un état de tension permanent et fécond entre acadienité et américanité. C'est effectivement dans cette dualité assumée ou imposée qu'elle trouve son originalité, en un mot son identité.

\title{
L'acadienité affective ou la marque de fabrique "nationale "d'une littérature francophone régionale
}

Nationale, la poésie acadienne l'est sans nul doute dans le sens qu'elle renvoie aux grands standards d'une acadienité affective, ancrée dans une histoire et sur un territoire. Être Acadien, c'est d'abord avoir conscience de soi, de son histoire et de sa culture particulière bercée par le catholicisme (Lamarque, SCHEC, 2008b). Les poètes acadiens ne dérogent pas à cette règle, même si cette évocation reste moins importante que dans la littérature du premier $X X^{\text {ème }}$ siècle. Pierre angulaire de leur destin, la tragédie du Grand Dérangement - leur déportation - revient plus ou moins directement sous leur plume. D'abord en tant qu'évènement historique, comme dans la description qu'en fait Glen Charles Landry dans son poème intitulé Déportation (2006), et qui n'est pas sans rappeler celle de Henri Wadsworth Longfellow dans Evangeline, lorsqu'il écrit:

\author{
Les enfants brulent dans nos champs, Marie \\ Les enfants crient dans nos maisons, Marie \\ Les enfants pleuvent dans nos yeux, Marie \\ Les enfants tombent à leurs morts, Marie \\ Nos enfants sont où, Marie? \\ Nos enfants sont où?
}

Plus que le Grand Dérangement en tant que tel, ce sont ses conséquences géographiques et psychologiques, aux fondements mêmes de ce que l'on pourrait appeler "mal-être acadien" qui sont constamment rappelées. La rhétorique de l'errance revient ainsi

\footnotetext{
${ }^{1}$ Anthologie de la poésie acadienne, Moncton, Editions Perce-Neige, 2009.
} 
en boucle dans la poésie acadienne. Elle acte non seulement la dispersion de cette diaspora à travers le monde, mais aussi la disparition à proprement parler de l'Acadie en tant que pays. Si Gérald LeBlanc dans son poème Peticodiac (1999) associe allégoriquement cette errance à celle des "bikers de la tendresse "sur une route asphaltée "pleine de trous"; Herménégilde Chiasson ressent plutôt "la misère de l'errance" dans son poème Amérique (1986); alors que Daniel Omer LeBlanc trouve dans le vagabondage l'image adéquate ${ }^{2}$.

Beaucoup d'entre eux - dans une démarche de simplification historique - reviennent sur les similitudes existantes avec la diaspora juive ${ }^{3}$. À l'errance, c'est-à-dire à la mobilité imposée, succède la notion contradictoire d'isolement, c'est-à-dire d'absence de liberté. Le prisme mémoriel est là encore très fort. Autorisés à revenir sur leurs terres à partir de 1763, les Acadiens ne peuvent occuper que les arpents les plus pauvres et les plus excentrés des nouvelles provinces créées par l'administration britannique. Leur retour s'associe donc à une forme d'ostracisme et de ségrégation géographique et sociale.

Raymond Guy LeBlanc dans son poème Archives de l'absence (2005) est très certainement celui qui a le mieux parlé de ce sentiment d'isolement, qu'il rapproche très adroitement des rigueurs climatiques. Le registre de la précarité est aussi très prégnant et reflète là encore autant un héritage qu'une actualité. Tout d'abord, il est historiquement établi que les communautés acadiennes ont été les populations les plus pauvres et les plus corvéables du Canada Atlantique (Landry / Lang, 2005). Leur retour s'est accompagné d'un flux de mendicité dans les principales villes anglophones de la région. Même si depuis les années 1960, leur situation s'améliore considérablement, il n'en demeure pas moins qu'un important écart de développement perdure toujours. Ronald Després reflète parfaitement ce ressenti lorsqu'il parle dans son poème Les mains (1975), des "mains rugueuses de l'ouvrier (...)" des "mains qui quêtent dans la rue" ou encore des "mains osseuses de la faim". Tout comme Georgette LeBlanc dans son poème Aux Etats (2006), qui raconte la vie d'une famille pauvre contrainte à l'exil.

De cette mémoire intégrée découlent deux réactions contradictoires. La première est mélancolique et se rapproche d'une certaine manière de la saudade portugaise (Eduardo Lourenço, 1978). Herménégilde Chiasson écrit par exemple dans son poème Eugénie Melanson (1974):

\section{(...) aujourd'hui vous êtes tous ici vous êtes emprisonnés, toi, les médailles du Vatican, le tableau de la déportation, le drapeau de lin que Monseigneur richard avait fait faire, et tous les rêves qui vivent derrière les vitres de cette grande}

\footnotetext{
${ }^{2}$ Le poème Vagabond dans son recueil: Omégaville, Moncton, Editions Perce-Neige, 2002.

${ }^{3}$ Notamment Gérald LeBlanc dans son poème Mouvance, dans le recueil Lieux transitoires, Moncton, Editions Perce-Neige, 1986.
} 
cage à nostalgie. [...]

La deuxième réaction très perceptible est celle de la révolte, conformément d'ailleurs au vent de contestation soufflant sur la société acadienne à partir des années 1968 et qui tranche avec plusieurs décennies de silence et de repli. Ronald Deprés rappelle ce sursaut dans son poème La nuit de la poésie acadienne (1975):

(...) tout à coup

un pays nu sans frisson

un pays de prunelles fières

et de poings tendus

vers la lumière.

tu es, mon Acadie

— et sans douleur, cette fois -

pays de partance. (...)

Plus provocant, Ulysse Landry se questionne dans son poème Peut être (1977):

(...) peut-être

faudrait-il mettre feu au pays

pour n'avoir plus à compter sur la bêtise du voisin

et peut-être pourrions-nous nous installer

sur des iles anonymes

et jouer à la poupée

et lui faire l'amour sous la silhouette du crucifix (...)

Gérald LeBlanc se fait, de son côté, plus catégorique dans son Eloge du Chiac (1995):

(...) nous ne voulons plus ressembler

à ceux qui nous acceptent

à condition que nous effacions

toute trace d'histoire personnelle

qui nous aiment à genoux

devant l'autel de l'aliénation

c'est même pus funny (...)

La remise en cause de cette situation historiquement imposée se double aussi d'une contestation des structures traditionnelles de la société acadienne et des relations sociales 
qu'elles induisent. Ronald Léger fait ainsi dans ses poèmes le procès d'une société trop paternaliste et trop ancrée dans la religion (Le miracle, 2003), tout en abordant des problèmes sociétaux comme l'alcoolisme (Le lavage, 2007).

Disparue en tant que telle depuis des siècles, la poésie acadienne reste pourtant attachée plus qu'une autre au territoire que constitue l'Acadie contemporaine. II semble même exister un lien indéfectible entre les auteurs et leur milieu géographique immédiat. En termes d'écriture cela se transcrit de deux façons. D'abord par des descriptions de type macroscopique, plutôt impersonnelles, invitant le lecteur à la curiosité ou le laissant luimême localiser par intuition les endroits suggérés.

C'est le cas par exemple du poème de Raymond Guy LeBlanc Cri de terre (2005), où l'auteur mêle astucieusement la description d'une nature chaotique avec la mélancolie acadienne. II peut ensuite s'agir de descriptions beaucoup plus précises, ancrant les poèmes dans une certaine réalité physique, comme par exemple le poème de Marc Arseneau Bury my heart à Beaumont (2002), dans lequel il écrit:

\author{
à beaumont une dernière fois \\ je regarde la marée monter la petitcodiac \\ la Memramcook et la Chipoudie \\ dis-moi pépére aujourd'hui \\ avant que leur hache me fendent le tchoeur \\ faut-tu que je watch cette belle place \\ devenir un autre Kouchibouguac \\ estropier mes rêves comme ça \\ avec une hache pis une chainsaw
}

L'évocation de l'Acadie rurale et littorale renoue quant à elle avec le mythe des origines - celui des premières paroisses acadiennes - tout en donnant un portrait très fidèle des paysages du Canada atlantique. Les champs lexicaux pélagiques dominent ainsi très largement les vers acadiens, où il est question de plages, de marées, de coquillages, de bateaux, de pécheurs, de galets, de goélands, de phares, de varech, à l'instar du poème emblématique de Mario Thériault La trilogie du sable (1992). Espace de rencontre entre terre et mer, le tropisme des marais, sur lesquels les Acadiens ont été obligés de s'installer à leur retour d'exil, est aussi une constante, comme dans le poème de Robert Pichette Miscou (1987) lorsqu'il évoque les "hérons bleus" dans "l'eau plate des marais à deux pas de la mer".

L'Acadie urbaine, qui est évoquée dans des proportions exactement identiques, fait quant à elle référence à une autre Acadie, celle de la modernité, celle d'aujourd'hui et celle de demain. Elle retranscrit à sa manière une réalité sociale: l'exode d'une partie de plus en 
plus importante des populations acadiennes vers les centres urbains, à la recherche d'une meilleure éducation et d'un niveau de vie supérieur. Aujourd'hui, plus d'Acadiens vivent effectivement dans des zones urbaines que dans les zones reculées des Provinces Maritimes. Moncton semble le plus exciter la plume des poètes acadiens. Depuis la fin des années 1960, cette bourgade est devenue la capitale culturelle de l'Acadie contemporaine, eu égard à son université de langue française et au consulat général de la France qui y est installé (Pichette, 1998).

Gérald LeBlanc divague ainsi sur sa traversée télépathique de Moncton dans son poème Mouvance (1986). Herménégilde Chiasson assiste quant à lui à la fuite de la figure mythologique d'Hypnos sur la rue Main ${ }^{4}$. Hélène Harbec décrit de son côté une scène improbable autour d'un goéland blessé dans la rue Clément Cormier dans son poème du même nom (2002). Qu'ils soient enfin ruraux ou urbains, la très nette majorité des lieux évoqués se situent dans la province canadienne du Nouveau-Brunswick, alors que les Acadiens du Canada sont éclatés sur au moins deux ou trois autres provinces. Cela reflète sans nul doute le dynamisme de la communauté acadienne de cette province, dont le poids démographique, mais aussi l'histoire politique récente (Cormier, 2004), lui confère une position d'aiguillon de la diaspora.

Plus que jamais donc la poésie acadienne des trente dernières années reste "nationale", plus ou moins enfermée dans son histoire chaotique et ses conséquences encore perceptibles; et charnellement attachée à son bout de terre aux confins orientaux de l'Amérique du Nord.

\section{L'américanité effective ou les influences incontournables d'un continent culturellement anglo-saxon}

Cosmopolite, la poésie acadienne le devient en contact avec l'américanité omniprésente au sein duquel elle évolue, perdant ainsi de ses saveurs et spécificités nationales. En lisant attentivement les poèmes acadiens, on se rend compte qu'ils reflètent l'appartenance de cette société à une aire continentale bien spécifique: celle de l'Amérique du Nord. Très caractéristique des littératures nord-américaines, les notions d'espace et de climat occupent à cet égard une place relativement prépondérante (Suberchicot, 2002).

L'Acadie s'inscrit effectivement dans un milieu naturel, où les éléments s'enchaînent et se déchaînent, façonnent autant que fascinent, renforcent autant que séparent les membres de cette communauté. La place faite aux saisons, et notamment les références à l'hiver, sont ainsi omniprésentes au point d'en devenir obsessionnelles. La virulence des

\footnotetext{
${ }^{4}$ Dans son poème Hypnos s'enfuit de la rue Main, lui-même extrait de son recueil Climats, Moncton, Editions de l'Acadie, 1996.
} 
hivers marque en effet dès les origines l'histoire du peuple acadien, puisqu'elle décime la première colonie établie en 1604. Profondément rigoureux en raison de la continentalité du climat, les hivers sont historiquement associés à des périodes de dangers autour desquelles toute la vie s'organise. Raymond Guy LeBlanc a raison lorsqu'il dit venir "d'un peuple qui sait l'hiver" et qui voit dans chaque printemps une espèce de "grande fête" (Archives de l'absence, 2005).

Pour Ronald Després, l'Acadie hivernale ressemble à "un pays transi et frileux, comme un hôtel des brumes" (Nuit de la poésie acadienne, 1975). Annick Perrot-Bishop évoque de son côté "la somnolence du ciel d'hiver" (Dimanche, 1996), et le "goût frais" de la neige qui a tendance à "ensevelir la mémoire" (Terre Neuve, 1996). Mais à ces évocations traditionnelles de l'hiver nord-américain succèdent aussi des conceptions plus joyeuses comme celles que l'on retrouve dans les vers d'Herménégilde Chiasson.

Bien plus qu'une saison, l'hiver est un partenaire obligé de la vie en Amérique, qui influe sur son organisation sociale et économique, mais aussi d'une certaine manière sur ses orientations intellectuelles. Proportionnellement, les autres saisons passent inaperçues ou sont toujours évoquées par rapport à la période hivernale comme dans Galvanie (2006) de Christian Brun ou encore Eugénie Melanson (1974) d'Herménégilde Chiasson et bien entendu dans La vie, le temps (2006) de Rino Morin Rossignol:

\footnotetext{
novembre peut bien gémir dans les arbres dépouillés, novembre peut bien prendre un air glacial et menacer toute âme qui vive d'un hiver sans scrupule et sans rémission, novembre peut bien tenter d'obscurcir la lumière du jour, n'être qu'un frisson gris et pluvieux, avant longtemps novembre passera, novembre ne sera plus qu'un désagréable souvenir, novembre s'éclipsera à jamais (...) irrémédiable automne devant l'arbitraire de la vie. Celle qui coule droit devant, celle qui sillonne derrière. le vent tourne, le vent se lève sur les flancs ravagés du désir et l'homme est encore chargé d'ambition et de promesses.
}

En cela une intertextualité existe entre la poésie acadienne et ses voisines canadiennes et américaines, du moins celles de la côte Est. On ressent l'incidence de la continentalité américaine dans le tropisme qu'exercent les grandes métropoles sur les auteurs acadiens. Première ville destinatoire des Acadiens quittant l'aire des Maritimes, Montréal semble la cité des possibles, la porte d'entrée vers l'autre Amérique, celle aussi des interdits transcendés, des amours passionnels, mais aussi des gestes quotidiens. Si Eric Cormier, dans une logique toute proustienne, associe l'une de ses pérégrinations dans la 
métropole montréalaise au souvenir de l'être aimé5; plus concrète France Daigle à la recherche de Marianne Godbout, nous donne l'occasion d'entamer un véritable parcours initiatique dans le Montréal populaire ${ }^{6}$. Ce tropisme urbain ne se limite pas simplement à cette ville, mais s'étend aussi à toutes les mégalopoles d'Amérique, et même d'ailleurs. Gérald LeBlanc écrit dans ses Visions de Rimbaud (1986):

j'entends à la radio que Rimbaud est revenu en ville.

et je me suis rappelé le temps passé ensemble, à l'époque où je fumais

beaucoup trop, entre la mescaline et le chèque de chômage (...)

parfois, nous descendions jusqu'au Cap-pelé où on passait l'après midi, les orteils dans le sable, à énumérer toutes les villes qui nous fascinaient: San Francisco, Barcelone, New Orléans, Tokyo. il avait un mot pour cette activité, il appelait ça: désir (...)

la dernière fois qu'on s'est vus, c'était à New York. nous avions traversé Central park comme ça, ne distinguant plus l'est de l'ouest. dans un fast-food au coin de la 46e et Lexington, il écrivit: Burger King is Murder. il avait même suggéré qu'on écrive un poème sur le code postal. une mémoire réciproque nous travaillait (...)

L'influence de la civilisation américaine, avant tout urbaine, est donc ici très perceptible, la poésie acadienne rejoignant une nouvelle fois les caractéristiques de sa grande voisine étatsunienne. Évoluant dans un milieu dit minoritaire, la société acadienne est aussi la fille d'un métissage des cultures françaises et anglaises. Cette interculturalité à la mode canadienne rejaillit très clairement dans la poésie acadienne, l'éloignant de ses racines nationales. Les références gastronomiques en sont l'un des meilleurs exemples.

Si Raymond Guy LeBlanc loue le bon "fricot du homard des ployes" (Le temps de dire, 2005); Hélène Harbec les recettes aux "cerises de terre" et "les physalis au sirop" (Millefeuilles au chocolat et physalis au sirop, 2002); Herménégilde Chiasson imagine quant à lui une scène dans un Tim Horton's de la rue Main à Moncton ${ }^{7}$; Gérald LeBlanc évoque de son côté la culture "fast food" de l'Amérique dans ses Visions de Rimbaud (1986); alors qu'Ulysse Landry trouve une dimension érotique à une bouteille de Pepsi ${ }^{8}$.

$\mathrm{Du}$ point de vue des références musicales, on retrouve une dualité identique. Si Raymond Guy LeBlanc se laisse bercer comme beaucoup d'autres "au rythme d'une gigue et des chansons de folklores" (Acadie, 2005), les sons des violons acadiens laissent très

\footnotetext{
${ }^{5}$ Dans son poème La fin de Montréal, lui-même extrait du recueil Coda, Moncton, Editions Perce-Neige, 2003.

${ }^{6}$ Dans son poème Sur les traces de Marianne Godbout, cordonnière et savetière, Éloizes, $n^{\circ} 4,1981$

7 Dans son poème Hypnos s'enfuit de la rue Main, op. cit.

${ }^{8}$ Dans son poème Je joue avec les mots, lui-même extrait du recueil Tabous aux épines de sang, Moncton,

Editions de l'Acadie, 1977.
} 
rapidement leur place à des musiques plus déjantées de Bob Dylan, dont France Daigle (Ecriture et américanité, 1985), Marc Poirier (Guy, 1993) ou encore Herménégilde Chiasson (Visions de Rimbaud, 1986) font références:

nous écoutions les mêmes disques. Bob Dylan: Blonde On Blonde surtout. si je m'arrêtais à une image sibylline d'une chanson, il disait: "analyse pas, laisse les voyelles te bercer dans leurs sonorités, laisse toi emporter par leur travail sur les consonnes."

Mais au-delà de ces occurrences littéraires, l'interculturalité se retrouve surtout dans le style même de l'écriture acadienne. Le recours au Chiac est ainsi une caractéristique de la poésie. Mélange vernaculaire de français et d'anglais, principalement parlé dans la région de Moncton et parmi les générations les plus jeunes, il s'agit d'un dialecte acadien moderne. II s'éloigne du français acadien, car il mélange des phrases en français et des expressions en anglais. Longtemps dénigré, il bénéficie d'une revalorisation depuis la publication du recueil de Gérald LeBlanc intitulé Eloge du Chiac en 1995. Ce dernier écrit dans son poème inaugural:

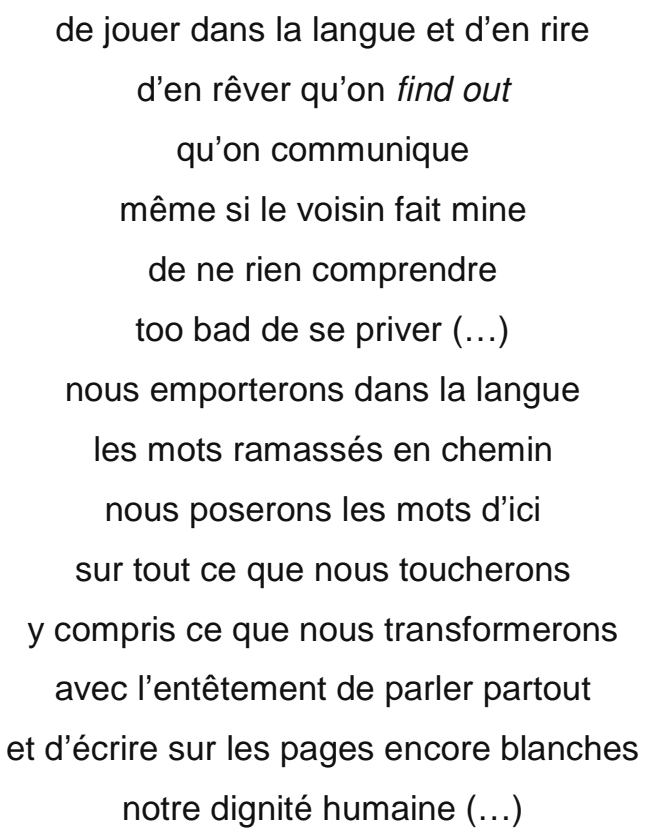

Bien plus qu'un snobisme littéraire ou intellectuel, le recours au Chiac apparaît sous la plume de Gérald LeBlanc être une forme de militantisme identitaire. Le renversement sémiotique est d'ailleurs particulièrement intéressant: alors que le Chiac pourrait légitimement être assimilé à un accident de l'histoire, voire une dégénérescence de la langue française dans ses réalités acadiennes; il devient le symbole d'une nouvelle acadienité 
métissée. Certains titres de poèmes en disent long sur ce mariage circonstancié entre le français et l'anglais comme Tableau de back yard (1994) de Guy Arsenault ou War chiac (1975) de Charles Landry, dont voici quelques extraits:

\author{
il arrive en gros plane, man! \\ Chassé par son propre crash landing \\ Right dans l'middle d'un désert de sable rouge. \\ Fuck man! Watch out pour la plow! \\ vois-tu pas son blinker
}

Plus que jamais, la poésie acadienne est une littérature d'influences et de confluences. Elle n'échappe pas au fond au contexte culturel à dominante anglophone dans lequel elle baigne quotidiennement. Elle nous rappelle surtout que les poètes acadiens, sont avant tout des Américains, certes de langue française, mais imprégnés par les grands items de la vie sur ce continent.

$\mathrm{Au}$ terme de cette réflexion par définition non exhaustive et volontairement simplificatrice, on peut donc dire que dans la problématique de ce colloque autour de la notion de "littérature nationale", la poésie acadienne est en réalité un genre parfaitement hybride. Elle appartient d'une part à la littérature identitaire, lorsqu'elle évoque les spécificités de son passé qui en font un peuple au destin unique dans le monde. Elle devient l'une des variantes régionales de la littérature francophone, lorsqu'elle décrit minutieusement le territoire auquel elle est attachée. Elle s'en éloigne enfin en devenant une "littérature monde" lorsqu'elle se laisse pénétrer par les influences de l'américanité.

Tout ceci pourrait faire légitimement croire à une poésie sans personnalité, dénuée véritablement d'ancrages, en un mot nomade. Mais si on y réfléchit bien, la poésie acadienne mérite bien plus qu'une autre, le qualificatif de "national" ou plus exactement d'identitaire. D'abord parce qu'elle reflète les réalités d'une communauté francophone confrontée quotidiennement aux défis de l'assimilation; mais aussi parce qu'elle matérialise l'âpreté d'un combat: celui de la survie et la reconnaissance d'une langue minoritaire. 


\section{Bibliographie}

\section{Généralités}

Cormier, Michel (2004). Louis Robichaud - La Révolution Acadienne, XXXX: Edition Lemeac.

LAMARQUE, Gwénael, “Une Acadie littéraire entre tradition et modernité à travers l'exemple des écrivains de l'association acadienne des artistes professionnel(le)s du Nouveau-Brunswick "(2008a). In: Intercâmbio, $2^{\mathrm{a}}$ série, nq1, pp.?

LAMARQUE, Gwénael, “L'Église catholique acteur de la francophonie en Amérique du Nord: l'exemple de la culture acadienne ". (2008b). In: Revue de la SCHEC, n\%4.

LANDRY, Nicolas / LANG, Nicole (2001). Histoire de l'Acadie. Québec: Les éditions du Septentrion / Sillery.

LOURENÇO, Eduardo (1978). Mythologie de la saudade. Paris: Editions Chandeigne.

MAGORD, André (2003). L’Acadie plurielle: dynamiques identitaires collectives et développement au sein des réalités acadiennes. Moncton-Poitiers, Centre d'études acadiennes et Institut d'études acadiennes et québécoises: Université de Moncton.

PICHETTE, Robert (1998). Napoléon III. L'Acadie et le Canada français. Moncton: Les Éditions d'Acadie.

SUBERCHICOT, Alain (2002). Littérature américaine et écologie. Paris: L'Harmattan.

\section{Anthologies}

LeBlanc, Gérald / Beausoleil, Claude (1999). Anthologie de la poésie acadienne. Moncton: Editions PerceNeige.

ThibodeAu, Serge Patrice (2009). Anthologie de la poésie acadienne. Moncton: Editions Perce-Neige.

\section{Recueils poétiques}

Arseneau, Marc (2002). Avec l'idée de l'écho. Moncton: Editions Perce-Neige.

BRUN, Christian. (2006). Tremplin. Moncton: Editions Perce-Neige.

CHIASSON, Herménégilde (1974). Mourir à Scoudouc. Moncton: Editions de l'Acadie.

CHIASSON, Herménégilde (1986). Prophéties. Editions Michel Henry.???

DAIGLE, France (1981). "Sur les traces de Marianne Godbout, cordonnière et savetière". In: Éloizes, $\mathrm{n}^{\circ} 4$.

DAIGLE, France (1985). Les cent lignes de notre américanité. Moncton: Editions de l'Acadie.

Despres, Ronald (1975). Paysages en contrebande à la frontière du songe. Moncton: Editions de l'Acadie.

HARBEC, Hélène (2002). Va. Moncton: Editions Perce-Neige.

LANDRY, Glen Charles (2006). Croquis urbain d'un Francorien. Moncton: Editions Perce-Neige.

LANDRY, Ulysse (1977). Tabous aux épines de sang. Moncton: Editions de l'Acadie.

LeBlanc, Georgette (2006). Alma. Moncton: Editions Perce-Neige.

LeBlanc, Gérald (1995). Eloge du Chiac. Moncton: Editions Perce-Neige.

LeBlanc, Gérald (1999). Je n'en connais pas la fin. Moncton: Editions Perce-Neige.

LeBlanc, Raymond Guy (2005). Archives de la présence. Moncton: Editions Perce-Neige.

LEGER, Ronald (2003). tachyAcadie. Moncton: Editions Perce-Neige.

LEGER, Ronald (2007). Les poissons s'arrêtent. Moncton: Editions Perce-Neige.

Perrot-Bishop, Annick (1996). Au bord des yeux de la nuit. Moncton: Editions de l'Acadie.

PICHETTE, Robert (1987). Béllérophon. Moncton: Editions de l'Acadie.

POIRIER, Marc (1993). Avant que tout disparaisse. Moncton: Editions Perce-Neige.

Rossignol, Rino Morin (2006). Intifada du cœur. Moncton: Editions Perce-Neige.

Theriault, Mario (1992). Echographie du Nord. Moncton: Editions Perce-Neige. 